\title{
The mechanical response of micron-sized molecular crystals
}

\author{
Christopher M. Barr ${ }^{1} \cdot$ Marcia Cooper ${ }^{1} \cdot$ Jeremy Lechman $^{1} \cdot$ Daniel C. Bufford ${ }^{1}[0$
}

Received: 4 June 2021 / Accepted: 11 August 2021 / Published online: 3 September 2021

(c) National Technology \& Engineering Solutions of Sandia, LLC 2021

\begin{abstract}
Microstructures and corresponding properties of compacted powders ultimately depend on the mechanical response of individual particles. In principle, computational simulations can predict the results of powder compaction processes, but the selection of appropriate models for both particle-particle interactions and particle deformations across all relevant length scales remain nontrivial tasks, especially in material systems lacking detailed mechanical property information. The work presented here addresses these issues by conducting uniaxial compressions in situ inside of a scanning electron microscope to characterize the mechanical response of individual micron-sized particles of a molecular crystal, hexanitrohexaazaisowurtzitane (CL-20). This experimental approach enabled the collection of quantitative force and displacement data alongside simultaneous imaging to capture morphology changes. The results reveal information about elastic deformation, yield, plastic deformation, creep, and fracture phenomena. Accordingly, this work demonstrates a generalizable approach for assessing the mechanical response of individual micron-sized molecular crystal particles and utilizing those responses in particle-level models.
\end{abstract}

\section{Introduction}

Granular solids form from agglomerations of discrete particles of essentially any material, and bulk properties emerge from both the properties of the particle constituents and the microstructures formed during packing. In principle, computational simulations can predict the results of quasistatic powder compaction, as in the discrete element method (DEM) or peridynamics [1-4]. However, realistic models must account for particle rearrangement, deformation, and fracture across all relevant length scales. Progress in this area demands detailed mechanical properties information for the individual particles, including both intrinsic material properties and effects of extrinsic factors like size and shape.

Molecular crystals find use in pharmaceutical, organic optoelectronic, and energetic materials applications, and frequently undergo deformation and fracture during processing and use. These materials consist of molecules bound together by intermolecular forces including hydrogen bonds, van der Waals forces, and $\pi$-interactions [5]. These bonding forces differ substantially from metallic, ionic, and

Daniel C. Bufford

dcbuffo@sandia.gov

1 Sandia National Laboratories, PO Box 5800, Albuquerque, NM 87185, USA covalent bonds in other solids, giving rise to a great variety of different mechanical properties [6]. The understanding of mechanical deformation and fracture in molecular crystals remains less mature than in other classes of materials [7]. While indentation-based methods have proven valuable for investigating strength and elasticity [8-17], such approaches provide less insight into constitutive stress-strain response, fracture, and extrinsic length scale- or shape-dependent changes in deformation behavior. Indentation fracture methods [18] have been utilized successfully in some cases, but high degrees of anisotropy generally lead to difficulties in applying these approaches to molecular crystals [16]. Little information is available regarding mechanical properties of molecular crystals with external dimensions at the micrometer scale and below, a size regime where deformation behaviors often deviate from the bulk in other materials [19]. A means to characterize deformation at such length scales is highly desirable but remains difficult to realize in practice.

Our experimental methods addressed these difficulties by employing a nanoindenter operated in situ inside of a scanning electron microscope (SEM) to execute uniaxial compression tests on individual micron-sized hexanitrohexaazaisowurtzitane (CL-20) particles with concurrent observation. Our results demonstrate both the utility and some of the challenges of this approach in evaluating deformation behaviors at small length scales in this material. Though 
irregular particle shapes complicated the interpretation of the initial contact regimes, the subsequent load-displacement responses to monotonic, cyclic, and constant loads, and fracture behaviors were more readily understood. The results suggest that smaller particles fractured at higher stresses than larger ones, an observation consistent with the "smaller is stronger" principle observed in many materials [20]. This work provides information that complements standard nanoindentation approaches for investigating fundamental deformation and fracture mechanisms, and demonstrates a means to provide more realistic input or validation for particle-based simulations.

\section{Experiment}

Important details of the experimental methods appear here, while the Supplementary Information contains a complete description. Samples were prepared from $\varepsilon$-CL-20 powder with a $2.6 \mu \mathrm{m}$ mean particle diameter by drop casting particles suspended in isopropanol onto a silicon wafer. Particle compression experiments utilized a Hysitron PI-85 nanoindenter [21] with nominal $6 \mu \mathrm{m}$ flat punch tip operated in situ inside a scanning electron microscope.

Figure 1 illustrates the geometry and viewing angle of a typical experiment. Load- and displacement-controlled experiments probed the viscoelastic response and fracture behaviors, respectively.

\section{Results}

An initial set of experiments scrutinized electron beam effects by repeatedly applying trapezoidal load functions to a single particle with the beam on and off. An overview of these experiments appears in the Supplementary Materials. Important results of these pilot experiments include:
1. Contact between the indenter and particle was only Hertzian for a brief period, hence the results are better interpreted as uniaxial compressions rather than contact experiments.

2. A small but consistent amount of creep deformation occurred during the constant-load hold segment.

3. Electron beam exposure lowered unloading stiffness values by $\approx 5 \%$, but loading and constant hold segments showed no noticeable effects.

Overall, these observations strongly suggest that this in situ method represents a valid approach for studying the mechanical response of these particles.

Fracture experiments utilized displacement-controlled mode with indenter displacement rates of $10-20 \mathrm{~nm} / \mathrm{s}$ and data acquisition rates of $200 \mathrm{~Hz}$. The tested particles ranged from 1.9 to $5.1 \mu \mathrm{m}$ in height, so first-order lower- and upper-bound estimates of the initial strain rate are $2 \times 10^{-3}$ to $1 \times 10^{-2} \mathrm{~s}$. Figure 2 presents a load-displacement curve with corresponding video snapshots illustrating a representative fracture experiment. Here, the load-displacement curve remained smooth until the indenter reached approximately $175 \mathrm{~nm}$ displacement, after which several successive small load drops occurred prior to the large load drop at point (2). The shaded area below the curve up to point (2) corresponds to the energy absorbed prior to the large load drop. Note that the indenter advances by means of ramping voltage on a transducer, so an active control loop adjusts voltage to maintain steady displacement control. In the event of a rapid change in a sample (e.g., fracture or burst of plasticity), the control loop must first detect the increase in displacement rate, then retract the indenter to the previous position before advancing at the prescribed rate again. Load drops thus typically correspond to rapid, discrete events associated with plasticity or fracture. The video (see Supplemental Materials) revealed no clear mechanism for the smaller load drops, but processes like dislocation bursts
Fig. 1 a SEM micrographs showing the indenter and sample surface at low magnification; $\mathbf{b}$ higher magnification view of the indenter tip nearly in contact with a single particle in preparation for a test
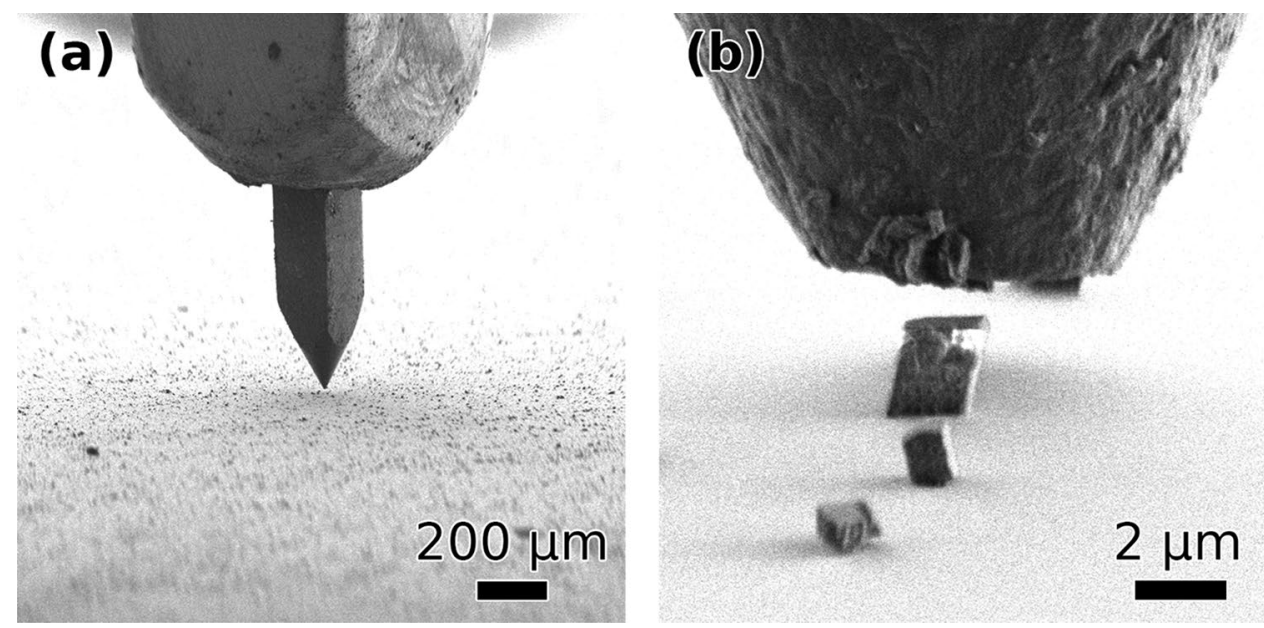
Fig. 2 a Load-displacement curve from a single particle in displacement control mode $(20 \mathrm{~nm} / \mathrm{s}$ up to $1000 \mathrm{~nm})$. The shaded area below the curve corresponds to the energy absorbed prior to the large fracture event at (2). Blue arrows in (a) show the path taken by the indenter during the excursion. Annotations (1-6) correspond to the images in the following panels. b (1-6) Sequence of images from the in situ SEM test. Orange arrow in (3) indicates the formation of a significant crack in the sample, and highlights additional crack opening in (5)

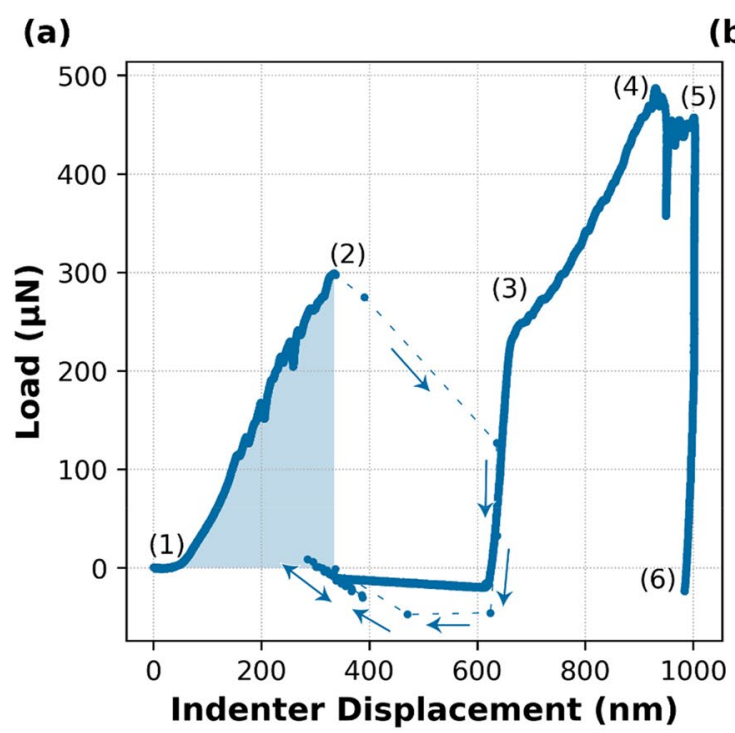

(b) (1)

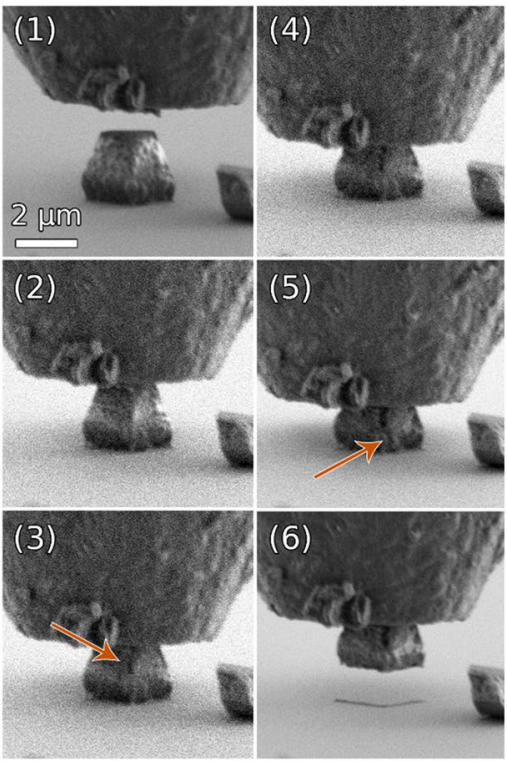

[21] or subcritical crack growth [22] have been implicated in other materials. The particle remained visibly intact prior to point (2) despite these events, an observation consistent with either plastic deformation or subcritical crack growth. In contrast, the large load drop at point (2) corresponded to a clear fracture event. The isolated data points between (2) and (3) in Fig. 2 capture the excursion; despite detecting the excursion and reacting within three data points $(15 \mathrm{~ms})$, the indenter essentially doubled the previously accumulated displacement before being retracted. The particle adhered to the indenter as it retracted, and no further deformation occurred until the particle was brought back into contact with the substrate. A comparison of Fig. 2b2, b3 illustrates the changes that occurred during this fracture event, specifically the appearance of a crack along the vertical edge of the particle, as denoted by the orange arrow. Additionally, the particle shortened along the compression axis and bulged in the perpendicular directions. After reestablishing contact, the load rapidly approached the $300 \mu \mathrm{N}$ value observed just before the initial large fracture event. As loading continued, the crack extended further downward midway between points (3) and (4), and culminated with another load drop between (4) and (5). A comparison of Fig. 2b4, b5 shows that the crack continued to open wider, and likely grew into the particle as well. The newer portion of the crack appeared to propagate in a different direction (downward and to the right) from the initial portion (downward and to the left). The direction change appeared to be correlated with the upper and lower facets of the particle, suggesting a relationship between the crack path and underlying crystal structure. Indenter motion briefly paused after reaching $1 \mu \mathrm{m}$ displacement, and the indenter was subsequently retracted to end the experiment. An examination of the load-time curve during the hold (see Supplementary Video SV1) revealed a smooth decrease in load over time during the pause, consistent with stress relaxation. While this behavior was not examined in detail, it provides additional qualitative evidence of viscoplasticity. The particle remained adhered to the indenter at the conclusion of the experiment, as shown in Fig. 2b6. During this experiment and similar replicates no deformation of the substrate beneath the particle was visible, and no motion of the substrate relative to the indenter was noted, indicating minimal frame compliance.

Thirty-seven similar replicates were conducted on other particles to investigate variability in fracture behavior. Numerous particles exhibited irregular morphologies, which caused significant difficulties in estimating contact areas, or caused issues like obvious shifting during loading. Hence, the fracture data presented in Fig. 4 represent eighteen total particles that yielded clean experiments that were practical for estimating fracture parameters. Here, the " $\times$ " symbol marks load drops that exceeded $25 \mu \mathrm{N}$ by prominence, which were identified using the "find_peaks" function implemented in Version 1.4.1 of SciPy [23]. Note that this threshold simply highlights the frequency of load drops, rather than indicating a well-defined fracture criterion. Still, each drop likely identifies a plastic deformation or fracture event. Interestingly, multiple small load drops appeared in some curves, while others showed none prior to the large drop associated with through-particle fracture. Some of the large fracture events consisted of single cracks similar to the one illustrated in Fig. 2, while others generated multiple cracks or crushed the particles outright as in Fig. 3. Despite the qualitative similarities, the curves varied considerably in how quickly the load rose, as well as in the number and extent of the load drop events. 
Fig. 3 Examples of two extremes of fracture behavior illustrated by initial and post-fracture micrographs. a, $\mathbf{b}$ Single through-particle crack. c, d Crushing and fragmentation. Scale bar is $2 \mu \mathrm{m}$ in all panels
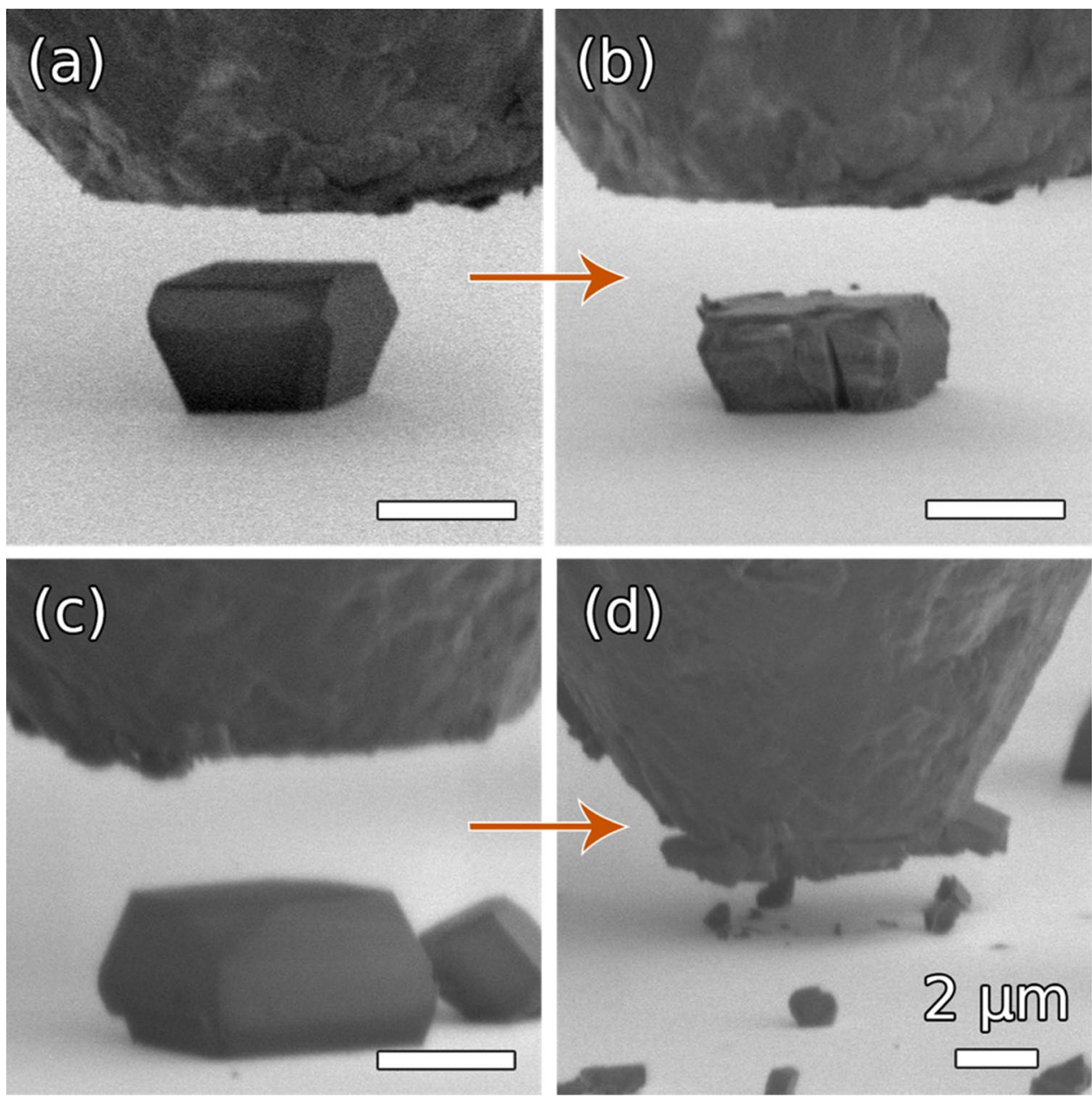
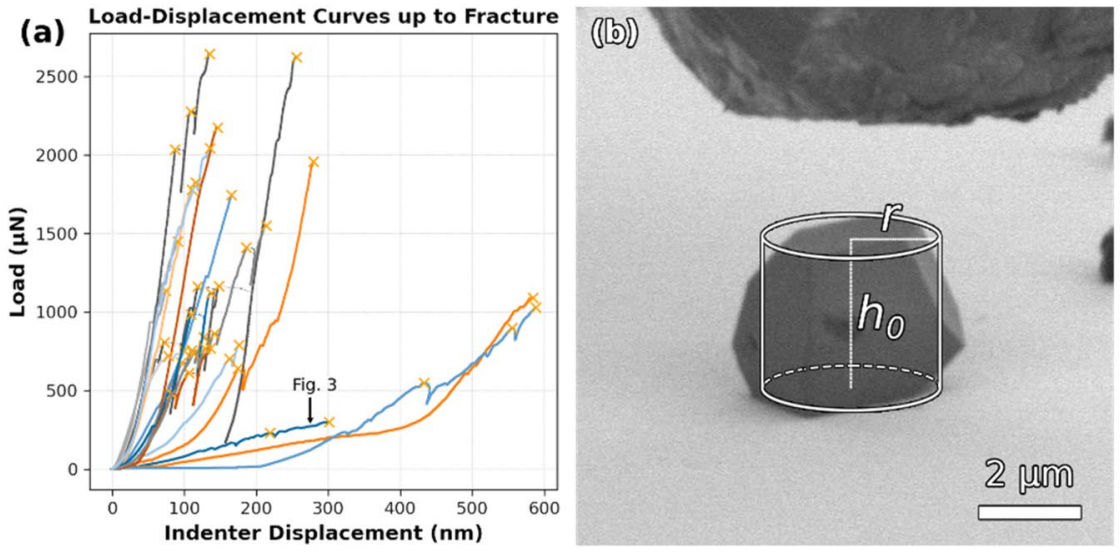

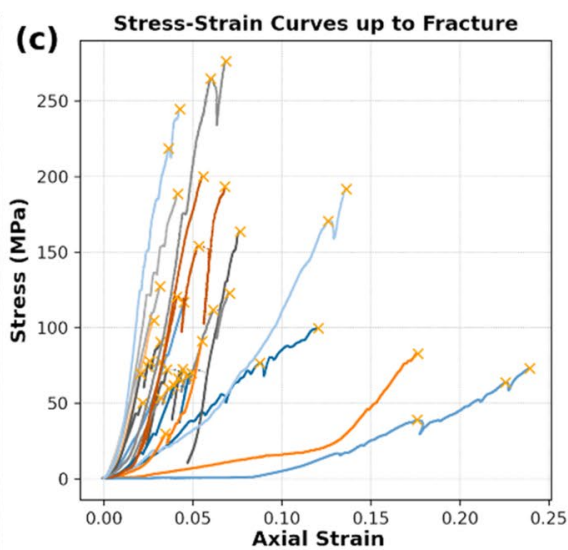

Fig. 4 a Variability in load-displacement behavior demonstrated by 18 load-displacement curves from single-particle compaction experiments. Load drops exceeding $25 \mu \mathrm{N}$ are marked by " $x$ " on each curve. The arrow and label denote the curve from Fig. 2 up to point

Variations in the initial loading behaviors point to variations in stiffness, which depends on both contact area and particle size. Accordingly, an effort was made to normalize
(2). b Illustration of the cylindrical geometry approximation used to compute stress and strain. c Engineering stress-strain curves computed from the load-displacement curves as described in the text

the load-displacement data into stress-strain curves, as in Fig. $4 \mathrm{~b}$ to better assess deformation and fracture behaviors. Initial width and height, $h_{0}$, of each particle in Fig. 4 was 
measured from the micrographs, and engineering strain was computed from the indenter displacement after contact was first made, $\delta$, by taking $\delta / h_{0}$ [24]. Particles were approximated as right cylinders with radius equal to one-half the measured width as illustrated schematically in Fig. 4b, and engineering stress was computed from the load data and the cross-sectional area of the cylinder. To account for error in these approximations, measurement uncertainty for the diameter and height have been conservatively estimated at $\pm 10 \%$ to accommodate particle shape irregularities, and uncertainty in the measured load has been conservatively estimated at $\pm 1 \mu \mathrm{N}$. The stress and strain estimates include substantial assumptions about the geometry of the particles and distribution of the applied loads; while the computed values may be inaccurate in the absolute sense, they are at least consistent among the particles examined here. The stress-strain curves in Fig. 4c collapse the data somewhat, which may suggest a consistent underlying constitutive relationship for the stress-strain response. Still, the curves vary substantially. Moreover, four curves show much greater plastic deformation prior to fracture than the rest. These outliers may stem from differences in crystallographic orientation, rather than just stochastic variation among similar particles.

\section{Discussion}

\section{Contact and elastic-to-plastic transition}

As noted in the Supplementary Materials, a Hertzian contact model does not appear appropriate beyond the first few $\mathrm{nm}$ after contact. Transitions from the initial contact to complete indenter-particle contact, then to yielding were variable and sometimes unclear. Most of the force-displacement curves from the fracture tests on other particles exhibited a smooth, qualitatively elastic loading behavior from the initial indenter contact up to yielding in the form of small load drops, while others showed a change in concavity prior to the appearance of load drops. The former may suggest an elastic response with temporally and spatially localized plasticity by discrete events (e.g., subcritical crack growth or dislocation bursts), whereas a gradual change in concavity suggests more continuous and widespread plasticity. Subtle yielding transitions have been described before in particles of other materials [25-27]. While the data presented here are not ideally suited for deeply scrutinizing the early stages of contact, they provide some insight. Nanoindentation experiments on flat surfaces may be able to probe the elastic-plastic transition more closely, particularly if spherical tip methodologies were used. Computational methods might also allow contacts to be scrutinized with arbitrary precision. In those cases, extensions of the Hertzian contact model that accommodate transitions to plastic deformation [25,
28-30] may provide adequate descriptions of local yielding for small deformations.

\section{Viscous flow}

Both load- and displacement-controlled experiments revealed some evidence of viscous flow in the form of creep and stress relaxation, respectively. Unfortunately, the SEM video could not resolve the questions regarding creep rate; videos were optimized to minimize electron dose, and as such as the resolution was around $15 \mathrm{~nm} /$ pixel, larger than the creep displacements themselves. Future work might provide additional clarity regarding viscous flow by varying loading rates, load hold magnitudes, and hold times, or by using higher magnification SEM video as a virtual strain gauge [31].

\section{Fracture}

As mentioned previously, the small load drops likely originated from phenomena associated with deformation or fracture. The lack of crystallographic orientation information precludes determining the mechanisms associated with these phenomena; however, some fracture parameters may be estimated from individual particle compaction data $[22,32]$. We focus on the large fracture event. As mentioned previously, the engineering stress-strain curves were computed by measuring particle dimensions and approximating the particles as cylinders. The resulting, stress, strain, and volumetric strain energy density at the point of fracture have been extracted from Fig. 4 and plotted as functions of the particle diameter in Fig. 5. These points are analogous to point (2) in Fig. 2a. Error bars in Fig. 5 reflect either the measurement uncertainty directly (estimated at $\pm 10 \%$ for length to accommodate particle shape irregularities and at $\pm 1 \mu \mathrm{N}$ for load) or propagation of error associated with computation of values from particle dimensions and load data.

Interestingly, the stress at fracture appeared to increase with decreasing particle diameter as seen in Fig. 5a. In contrast, engineering strain at fracture remained more consistent with no clear dependence on particle diameter. The largest strains at fracture arose from the outliers noted in Fig. 4, in which cases the underlying deformation processes likely differed from the rest of the population. Strain energy density provides a measure of the energy stored within a material undergoing deformation. Strain energy is the area under the stress-strain curve at a given point, computed here by numerically integrating the stress-strain curve up to the strain at fracture, i.e., $\int_{0}^{\varepsilon_{\text {fracture }}} \sigma d \varepsilon$, and the strain energy density was then computed by dividing by particle volume. Strain energy density at fracture also increased with decreasing particle diameter. The trends of varying strength and fracture behavior with particle diameter bear similarities to 
(a)

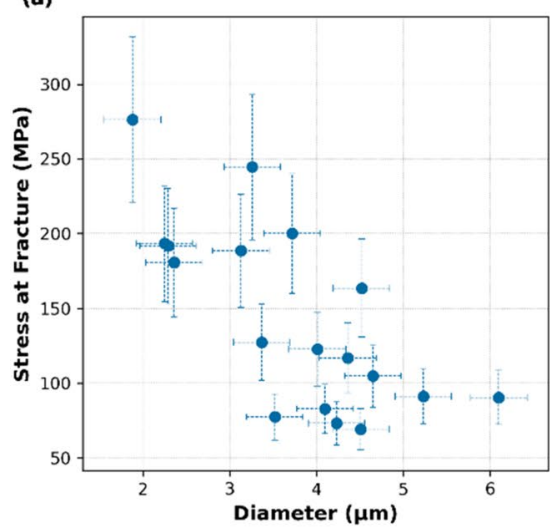

(b)

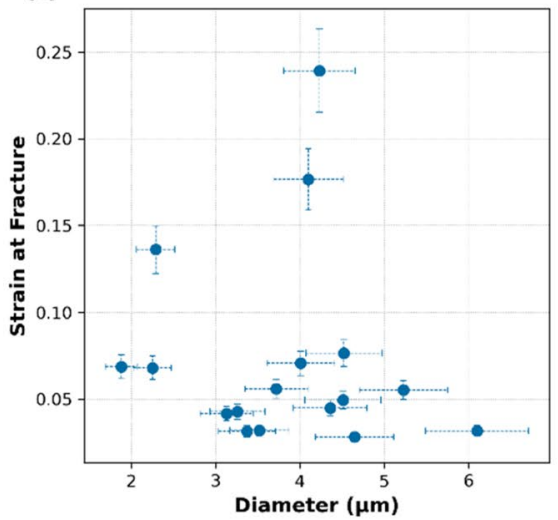

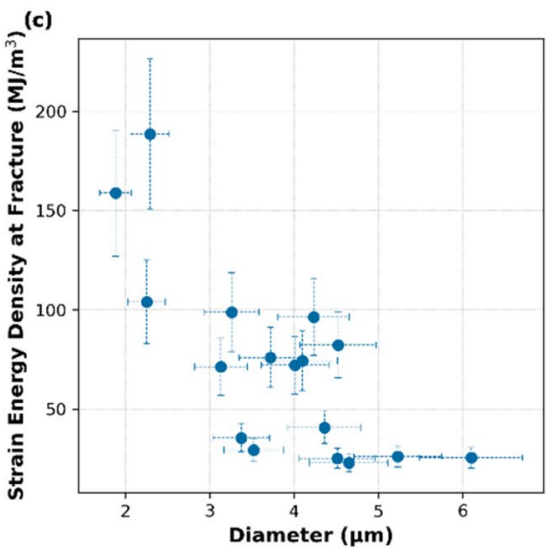

Fig. 5 Estimated a engineering stress, $\mathbf{b}$ engineering strain, and c strain energy density at fracture as a function of particle diameter for the experiments in Fig. 4. Error bars reflect $\pm 10 \%$ uncertainty in dimensional measurements, $\pm 1 \mu \mathrm{N}$ in the load measurement, and propagation of error for computed parameters

path. Particles like those illustrated in Figs. 2 and 3a, b fractured cleanly into two new pieces near the center of the particle, in which case the two new surface surfaces can be approximated as planes of area $2 r \times h_{0}$. On the other hand, some particles shattered into many pieces upon fracture, as in the extreme case illustrated by Fig. 3c, d. Here, the estimation of newly created surfaces becomes nontrivial. It is reasonable to assume that new surface area might be ten or more times larger than the single through-particle crack case. Accordingly, estimates for the crack area were made from the post-fracture micrographs for each particle in Fig. 4, with each particle being assigned either a single crack or "many" crack designation with ten times higher area. $G$ provides a complete description of fracture only in brittle materials, where all released strain energy creates new fracture surfaces. Plastic deformation as in Figs. 2 and 3 indicates that strain energy was partitioned between both the creation of fracture surfaces and plastic deformation. Continuing with the assumption of a brittle fracture process likely overestimates $G$, but will also provide an upper-bound. Under this assumption and that of either a single crack or multiple cracks totaling ten times the surface area of a single crack, we find $G$ in the range of 0.004 to $0.16 \mathrm{~kJ} / \mathrm{m}^{2}$, respectively. The outliers in Fig. 4 had values of $G$ that were larger than the rest of the population by a factor of 3 to 5 . The others averaged $0.012 \pm 0.009 \mathrm{~kJ} / \mathrm{m}^{2}$, which may be more realistic. For context, representative values of $G$ for silica glass [37], polystyrene [38], and 7000-series aluminum alloys [39, $40]$ are $\sim 0.004,0.01$, and $15 \mathrm{~kJ} / \mathrm{m}^{2}$, respectively. The critical stress intensity, $K_{\mathrm{IC}}$, is related to $G$ by $K_{\mathrm{IC}}^{2}=\mathrm{EG}\left(1-v^{2}\right)$. Using values of $18 \mathrm{GPa}$ and Poisson's ratio $v=0.21$ for CL-20 [41], we find $K_{\mathrm{IC}}$ ranging from 0.3 to $1.7 \mathrm{MPa} \sqrt{m}$. Again, the outliers in Fig. 4 contributed to significantly larger values: the others averaged $0.43 \pm 0.15 \mathrm{MPa} \sqrt{m}$. For context, $K_{\text {IC }}$ values for silica glass [37], polystyrene [38], graphs or computed with the assumption of a specified crack 
and 7000-series aluminum alloys $[39,40]$ are $\sim 0.8,3$, and $30 \mathrm{MPa} \sqrt{m}$. These values of $G$ and $K_{\mathrm{IC}}$ place CL-20 among brittle materials like silica glass and polystyrene. Little information regarding fracture toughness of CL-20 is available in the literature, but many other molecular crystals are brittle, with $K_{\mathrm{IC}}<1 \mathrm{MPa} \sqrt{m}$. One apt comparison might be to cyclotetramethylene tetranitramine (HMX) with $K_{\mathrm{IC}}$ of $\approx 0.25 \mathrm{MPa} \sqrt{m}$ estimated from nanoindentation fracture experiments [16].

These estimates of $G$ and $K_{\mathrm{IC}}$ should be treated with caution; the experiments were far from optimal for precisely assessing fracture parameters. The considerable variance in $G$ and $K_{\mathrm{IC}}$ stems from the dramatically different deformation and fracture behaviors among the tested particles, as illustrated in Fig. 3. As mentioned previously, there is likely some crystallographic orientation dependence on the deformation and fracture mechanisms that gives rise to these differences. We also note that the particles were tested in compression, and brittle fracture requires a tensile stress. Qualitatively, a particle develops internal tensile stresses as it barrels in response to compression, but a quantitative assessment of those stresses requires a good understanding of the relationship between applied stress and internal stresses. While in some cases the relationship may be well-characterized (e.g., the elastic field of a Hertzian contact between two convex bodies), here the irregular contacts and particle shapes pose a challenge for a quantitative assessment of internal stresses. The test geometry and lack of initial cracks prohibited the observation of steady crack growth, which is the preferred method of assessing crack growth behavior. While $K_{\mathrm{IC}}$ is generally considered a material property in the bulk, $K_{\mathrm{IC}}$ values rise and fall with decreasing length scale as plane strain to plane stress regimes are traversed, and conflicting observations have been reported regarding fracture at small length scales [36, $42,43]$. Thus, it bears stressing that the information here is relevant to fracture at this length scale and geometry, but may differ at other length scales and experimental setups. Still, these observations point to interesting unknowns in small-scale fracture of molecular crystals and demonstrate the necessary ingredients to assess fracture toughness at the micrometer scale. Future improvements to sample preparation could likely allow for the collection of more accurate fracture parameters.

\section{Conclusions}

While challenging from both the perspectives of sample preparation and experiment execution, small-scale mechanical deformation experiments performed in situ in electron microscopes comprise an active and exciting research area that has contributed substantially to the understanding of deformation mechanisms in many materials [36]. The work presented here demonstrates the feasibility of applying these methods to molecular crystals by characterizing the uniaxial compression responses of individual micron-sized CL-20 particles. Refinements to the sample preparation techniques in future work, for instance, micro- or nanoscale mechanical test structures fabricated from single crystals by focused ion beam (FIB) milling at either ambient or cryogenic temperatures, may reduce uncertainty in stresses and strains and provide greater clarity regarding crystallographic effects. The in situ experimental approach presented here provides complimentary information to ex situ nanoindentation, and combinations of both techniques with coordinated modeling will likely improve the understanding of the mechanisms that govern deformation and fracture in molecular crystals. Similar approaches could also be readily extended to probe timedependent deformation behaviors (i.e., creep, stress relaxation, and fatigue) that remain little-explored in molecular crystals [13, 44, 45].

Particle behaviors observed in this work spanned elastic deformation, yield, plastic deformation, creep, and fracture regimes-all scientifically interesting and relevant to industrial powder processing. The physical mechanisms that underly the observed deformation and fracture behaviors will remain an active area of investigation for some time to come. Still, the behaviors reported here could be incorporated within particle-based simulations in the near term. Such simulation tools may streamline the development of milling and compaction process development that currently rely heavily on empirical testing.

Supplementary Information The online version contains supplementary material available at https://doi.org/10.1557/s43580-021-00124-y.

Acknowledgments Amy Allen, Dan Bolintineanu, Brad Boyce, Joel Clemmer, Stacy Guo, Khalid Hattar, Nathan Heckman, Robert Knepper, Chad Macziewski, Michael Marquez, William Mook, Erick Ortiz, M. Barry Ritchey, David Rosenberg, Adam Sapp, Stewart Silling, and Alex Tappan (Sandia National Laboratories). Support provided by Sandia National Laboratories Laboratory Directed Research and Development (LDRD). This work was performed, in part, at the Center for Integrated Nanotechnologies, an Office of Science User Facility operated for the U.S. Department of Energy (DOE) Office of Science. Sandia National Laboratories is a multimission laboratory managed and operated by National Technology \& Engineering Solutions of Sandia, LLC, a wholly owned subsidiary of Honeywell International, Inc., for the U.S. DOE's National Nuclear Security Administration under contract DE-NA-0003525. The views expressed in the article do not necessarily represent the views of the U.S. DOE or the United States Government.

Data availability The datasets generated and analyzed in this study are available from the corresponding author on reasonable request.

\section{Declarations}

Conflict of interest The authors declare no conflicts of interest. 
Open Access This article is licensed under a Creative Commons Attribution 4.0 International License, which permits use, sharing, adaptation, distribution and reproduction in any medium or format, as long as you give appropriate credit to the original author(s) and the source, provide a link to the Creative Commons licence, and indicate if changes were made. The images or other third party material in this article are included in the article's Creative Commons licence, unless indicated otherwise in a credit line to the material. If material is not included in the article's Creative Commons licence and your intended use is not permitted by statutory regulation or exceeds the permitted use, you will need to obtain permission directly from the copyright holder. To view a copy of this licence, visit http://creativecommons.org/licenses/by/4.0/.

\section{References}

1. C.L. Martin, D. Bouvard, S. Shima, J. Mech. Phys. Solids. 51(4), 667-693 (2003). https://doi.org/10.1016/S0022-5096(02)00101-1

2. S.A. Silling, E. Askari, Comput. Struct. 83(17), 1526-1535 (2005). https://doi.org/10.1016/j.compstruc.2004.11.026

3. H. Kruggel-Emden, E. Simsek, S. Rickelt, S. Wirtz, V. Scherer, Powder Technol. 171(3), 157-173 (2007). https://doi.org/10. 1016/j.powtec.2006.10.004

4. M.L. Parks, R.B. Lehoucq, S.J. Plimpton, S.A. Silling, Comput. Phys. Commun. 179(11), 777-783 (2008). https://doi.org/10. 1016/j.cpc.2008.06.011

5. G.R. Desiraju, J. Am. Chem. Soc. 135(27), 9952-9967 (2013). https://doi.org/10.1021/ja403264c

6. C.M. Reddy, G.R. Krishna, S. Ghosh, CrystEngComm 12(8), 2296-2314 (2010). https://doi.org/10.1039/C003466E

7. I.A. Olson, A.G. Shtukenberg, B. Kahr, M.D. Ward, Rep Prog Phys. 81(9), 096501 (2018). https://doi.org/10.1088/1361-6633/ aac303

8. H.G. Gallagher, P.J. Halfpenny, J.C. Miller, J.N. Sherwood, D. Tabor, Philos. Trans. R. Soc. A 339(1654), 293-303 (1992). https://doi.org/10.1098/rsta.1992.0036

9. K.J. Ramos, D.E. Hooks, D.F. Bahr, Philos. Mag. 89(27), 23812402 (2009). https://doi.org/10.1080/14786430903120335

10. S. Varughese, M.S.R.N. Kiran, U. Ramamurty, G.R. Desiraju, Angew. Chem. Int. Edit. 52(10), 2701-2712 (2013). https://doi. org/10.1002/anie.201205002

11. M. Zhai, G.B. McKenna, Cryst. Res. Technol. 51(7), 414-427 (2016). https://doi.org/10.1002/crat.201500301

12. P. Manimunda, E. Hintsala, S. Asif, M.K. Mishra, JOM. 69(1), 57-63 (2017). https://doi.org/10.1007/s11837-016-2169-6

13. S. Mannepalli, S.K. Mangalampalli, Crystal. (2017). https://doi. org/10.3390/cryst7110324

14. M.R. Taw, D.F. Bahr, Prop. Expl. Pyro. 42(6), 659-664 (2017). https://doi.org/10.1002/prep.201600143

15. M.R. Taw, J.D. Yeager, D.E. Hooks, T.M. Carvajal, D.F. Bahr, J. Mater. Res. 32(14), 2728-2737 (2017). https://doi.org/10.1557/ jmr.2017.219

16. A.C. Burch, J.D. Yeager, D.F. Bahr, J. Mater. Res. 34(23), 3954 3963 (2019). https://doi.org/10.1557/jmr.2019.345

17. C. Wang, C.C. Sun, CrystEngComm 22(7), 1149-1153 (2020). https://doi.org/10.1039/C9CE01874C

18. G.R. Anstis, P. Chantikul, B.R. Lawn, D.B. Marshall, J. Am. Ceram. Soc. 64(9), 533-538 (1981). https://doi.org/10.1111/j. 1151-2916.1981.tb10320.x

19. J.R. Greer, J.T.M. De Hosson, Prog. Mater. Sci. 56(6), 654-724 (2011). https://doi.org/10.1016/j.pmatsci.2011.01.005

20. M.D. Uchic, D.M. Dimiduk, J.N. Florando, W.D. Nix, Science 305(5686), 986 (2004). https://doi.org/10.1126/science.1098993
21. K.A. Rzepiejewska-Malyska, G. Buerki, J. Michler, R.C. Major, E. Cyrankowski, S.A.S. Asif et al., J. Mater. Res. 23(7), 1973-1979 (2008). https://doi.org/10.1557/JMR.2008.0240

22. W.M. Mook, J.D. Nowak, C.R. Perrey, C.B. Carter, R. Mukherjee, S.L. Girshick et al., Phys. Rev. B 75(21), 214112 (2007). https:// doi.org/10.1103/PhysRevB.75.214112

23. P. Virtanen, R. Gommers, T.E. Oliphant, M. Haberland, T. Reddy, D. Cournapeau et al., Nat. Methods 17(3), 261-272 (2020). https://doi.org/10.1038/s41592-019-0686-2

24. M.A. Meyers, K.K. Chawla, Mechanical Behavior of Materials, 2nd edn. (Cambridge University Press, New York, 2009), p. 73

25. C. Thornton, K.K. Yin, Powder Technol. 65(1), 153-166 (1991). https://doi.org/10.1016/0032-5910(91)80178-L

26. L. Gilson, S. Kozhar, S. Antonyuk, U. Bröckel, S. Heinrich, Granul. Matter. 16(3), 313-326 (2014). https://doi.org/10.1007/ s10035-013-0464-2

27. A. Russell, P. Müller, J. Tomas, Chem. Eng. Sci. (2014). https:// doi.org/10.1016/j.ces.2014.04.016

28. L. Kogut, I. Etsion, J. Appl. Mech. 69(5), 657-662 (2002). https:// doi.org/10.1115/1.1490373

29. M.R. Brake, Int. J. Solids Struct. 49(22), 3129-3141 (2012). https://doi.org/10.1016/j.ijsolstr.2012.06.013

30. M.R.W. Brake, Int. J. Solids Struct. (2015). https://doi.org/10. 1016/j.ijsolstr.2015.02.018

31. D.C. Bufford, C.M. Barr, B. Wang, K. Hattar, A. Haque, JOM 71(10), 3350-3357 (2019). https://doi.org/10.1007/ s11837-019-03701-7

32. P. Sarobol, M. Chandross, J.D. Carroll, W.M. Mook, D.C. Bufford, B.L. Boyce et al., J. Therm. Spray Tech. 25(1-2), 82-93 (2016). https://doi.org/10.1007/s11666-015-0295-2

33. M.D. Uchic, D.M. Dimiduk, Mater. Sci. Eng. A 400-401, 268278 (2005). https://doi.org/10.1016/j.msea.2005.03.082

34. S. Yashima, Y. Kanda, S. Sano, Powder Technol. 51(3), 277-282 (1987). https://doi.org/10.1016/0032-5910(87)80030-X

35. Y. Jiang, F. Alonso-Marroquín, H.J. Herrmann, P. Mora, Granul Matter. 22(3), 69 (2020). https://doi.org/10.1007/ s10035-020-01038-6

36. G. Dehm, B.N. Jaya, R. Raghavan, C. Kirchlechner, Acta Mater. (2018). https://doi.org/10.1016/j.actamat.2017.06.019

37. S.M. Wiederhorn, J. Am. Ceram. Soc. 52(2), 99-105 (1969). https://doi.org/10.1111/j.1151-2916.1969.tb13350.x

38. G. Marshall, L. Culver, J. Williams, Int. J. Fracture. 9(3), 295-309 (1973). https://doi.org/10.1007/BF00049197

39. G. Hahn, A. Rosenfield, Metall. Trans. A 6(4), 653-668 (1975). https://doi.org/10.1007/BF02672285

40. N.U. Deshpande, A.M. Gokhale, D.K. Denzer, J. Liu, Metall. Mater. Trans. A 29(4), 1191-1201 (1998). https://doi.org/10.1007/ s11661-998-0246-3

41. X.J. Xu, H.M. Xiao, J.J. Xiao, W. Zhu, H. Huang, J.S. Li, J. Phys. Chem. B 110(14), 7203-7207 (2006). https://doi.org/10.1021/ jp060077v

42. A.R. Beaber, J.D. Nowak, O. Ugurlu, W.M. Mook, S.L. Girshick, R. Ballarini et al., Philos. Mag. 91(7-9), 1179-1189 (2011). https://doi.org/10.1080/14786435.2010.487474

43. T. Sumigawa, S. Ashida, S. Tanaka, K. Sanada, T. Kitamura, Eng. Fract. Mech. (2015). https://doi.org/10.1016/j.engfracmech.2015. 05.054

44. J.M. Katz, I.S. Buckner, Int. J. Pharmaceut. 442(1), 13-19 (2013). https://doi.org/10.1016/j.ijpharm.2012.09.006

45. D. Raut, M.S.R.N. Kiran, M.K. Mishra, A.M. Asiri, U. Ramamurty, CrystEngComm 18(20), 3551-3555 (2016). https://doi.org/ 10.1039/C6CE00575F 